\title{
On the principle of limiting amplitude
}

\author{
By
}

\section{Nobuhisa IwaSAKI*}

\section{§. Introduction and theorem}

We study the behavior for large time of solutions of wave equations with a harmonic forcing term in the three dimensional euclidean space and we prove the so-called limiting amplitude principle. The principle states that every solution $u(x, t)$ for the initial value problem,

$$
\begin{gathered}
\left\{\frac{\partial^{2}}{\partial t^{2}}+b(x) \frac{\partial}{\partial t}-\Delta+c(x)\right\} u(x, t)=f(x) e^{i \omega t} \\
\left.u(x, t)\right|_{t=0}=\left.\frac{\partial}{\partial t} u(x, t)\right|_{t=0}=0
\end{gathered}
$$

tends to the steady state solution, $e^{i \omega t} v(x, i \omega)$ uniformly on bounded sets at $t \rightarrow \infty$. Here $v(x, i \omega)$ satisfies the elliptic equation,

$$
\left\{-\Delta+c(x)+i \omega b(x)-\omega^{2}\right\} v(x, i \omega)=f(x) ;
$$

and the Sommerfeld radiation conditions at infinity. $\Delta$ denotes the Laplacian in $E^{3}$ and $\omega$ is a real number. In the case when $b(x) \equiv 0$ and the real valued function $c(x)$ is once continuously differentiable and its support is compact, this principle has been proved by $\mathrm{O}$. A. Ladyzenskaja [1]. Here the rate of approach to steady state is like $e^{-\varepsilon t}, \varepsilon>0$ as $t \rightarrow \infty$. When $b(x)$ and $c(x)$ satisfy that $b(x) \geq 0$, $b(x)=0\left(\frac{1}{|x|^{3+\varepsilon}}\right), c(x)=0\left(\frac{1}{|x|^{2+\varepsilon}}\right)$ as $|x| \rightarrow \infty$, and others, S. Mizohata and K. Mochizuki [2] had shown the principle, but they did not give the rate of approach. In this paper we shall obtain the rate $e^{-\varepsilon t}$ under the assumption that the real valued functions $b(x) \geq 0, c(x) \geq 0$ are bounded and their supports are compact.

Received October 30, 1967.

Communicated by S. Matsuura.

* Mathematical Institute. Faculty of Science, Kyoto University. 
Theorem 1. Let $f(x), b(x)$ and $c(x)$ be functions which satisfy the following conditions,

i) $f(x), b(x)$ and $c(x)$ vanish outside a bounded set

ii) $\sum_{\alpha \mid \leq 2}\left|D^{\alpha} f\right| \in L^{2}\left(E^{3}\right)$

iii) $\quad b(x) \geq 0, c(x) \geq 0$, and they are bounded functions.

And let $u(x, t)$ be a solution for initial value problem (1.1), (1.2). Then there exists a steady state $e^{i \omega t} v(x)$, such that

$$
\max _{x \equiv K}\left|u(x, t)-v(x) e^{i \omega t}\right| \leq C \cdot e^{-\varepsilon t}, \quad \exists \varepsilon>0, \text { as } t \rightarrow \infty,
$$

and $v(x)$ is a solution of (1.3) satisfying the Sommerfeld radiation conditions at infinity, that is,

$$
\int_{x_{1}=R}|u|^{2} d s=0(1), \int_{\mid x_{1}=R}\left|\frac{d}{d|x|} u+i \omega u\right|^{2} d s=o(1) \text { as } R \rightarrow \infty
$$

where $K$ is a bounded set of $E^{3}$.

We can regard a solution $u(x, t)$ as a twice continuously differentiable function $u(t)$ from $[0, \infty)$ to $L^{2}\left(E^{3}\right)$ and as a continuous function to $\mathscr{D}_{L^{2}}^{2}\left(E^{3}\right)$. In this sence there exists the unique solution of (1.1), (1.2) if $f(x) \in \mathscr{D}_{L^{2}}^{1}\left(E^{3}\right)$. Let $\tilde{u}(\lambda)$ be the Laplace image of $u(t)$ with respect to $t$,

$$
\tilde{u}(\lambda)=\int_{0}^{\infty} e^{-\lambda t} u(t) d t \text { in } L^{2}
$$

Then

$$
\tilde{u}(\lambda)=v(\lambda) / \lambda-i w
$$

and

$$
u(t)=\frac{1}{2 \pi i} \lim _{\tau \rightarrow \infty} \int_{\sigma-i \tau}^{\sigma+i \tau} \frac{v(\lambda)}{\lambda-i \omega} e^{\lambda t} d \lambda \text { in } L^{2}
$$

for large $\sigma>0$. Where

$$
\left\{-\Delta+c(x)+\lambda b(x)+\lambda^{2}\right\} v(\lambda)=f(x), v(\lambda) \in L^{2}, \operatorname{Re} \lambda>0 .
$$

(1.7) has the unique solution belonging to $L^{2}\left(E^{3}\right)$ if $f(x)$ belongs to $L^{2}\left(E^{3}\right)$ and $\operatorname{Re} \lambda$ is sufficiently large positive. Therefore we study the analyticity of $v(\lambda)$ with respect to $\lambda$ and the order of $\|v(\lambda)\|_{L^{2}(K)}$ as $|\operatorname{Im} \lambda| \rightarrow \infty$. 


\section{§. Some lemmas}

1) In the case when $b(x) \equiv c(x) \equiv 0$

$$
\left(-\Delta+\lambda^{2}\right) v(x, \lambda)=f(x), \quad f(x) \in L^{2}
$$

has the unique solution $v(x, \lambda)$ in $\mathscr{D}_{L 2}^{2}$ at $\operatorname{Re} \lambda>0$ and $v(x, \lambda)$ is an analytic function of $\lambda$ to $L^{2} . \quad v(x, \lambda) \equiv R(\lambda) f$ is represented by a fundamental solutions $E(\lambda)$ as following

$$
R(\lambda) f=E(\lambda) * f, \text { where } E(\lambda)=\overline{\mathscr{E}}\left\{\frac{1}{\left(4 \pi^{2}|\xi|^{2}+\lambda^{2}\right)}\right\}=\frac{e^{-\lambda|x|}}{4 \pi|x|} .
$$

Let $Q(\delta)$ denote a Hilbert space consisting of all functions $f$ such that $e^{\delta|x|} f \in L^{2}\left(E^{3}\right)$ with the inner product $(f, g)_{\delta}=\left(e^{\delta, x \mid} f, e^{\delta|x|} g\right)_{L^{2}\left(E^{3}\right)}$, $(-\infty<\delta<+\infty)$. Now it is clear that $Q(\delta) \subset Q\left(\delta^{\prime}\right)$ if $\delta \geq \delta^{\prime}$. Using these space,

Lemma 1. Let

$$
R(\lambda) f=\frac{1}{4 \pi} \int_{E^{3}} \frac{e^{-\lambda_{1} x-y \mid}}{|x-y|} f(y) d y .
$$

Then $R(\lambda)$, which values a bounded operator from $Q(2 \delta)$ to $Q(-2 \delta)$, is an analytic function of $\lambda$ and satisfies the following estimates at $\operatorname{Re} \lambda \geq-\delta(\delta>0)$.

$$
\begin{aligned}
\text { i) } & |R(\lambda) f|_{-2 \delta} \leq\left\{C(\delta) /(1+|\lambda|)\left(1+\left|R_{e}\right|\right)\right\} \cdot|f|_{2 \delta} \\
\text { ii) } & |D R(\lambda) f|_{-2 \delta} \leq\left\{C(\delta) /\left(1+\left|R_{e} \lambda\right|\right)\right\}|f|_{2 \delta} \\
\text { iii) } & \left|D^{2} R(\lambda) f\right|_{-2 \delta} \leq\left\{C(\delta)(1+|\lambda|) /\left(1+\left|R_{e} \lambda\right|\right)\right\}|f|_{2 \delta} \\
\text { iv) } & \mid R(\lambda)-R(\lambda+h)\}\left.f\right|_{-2 \delta} \leq\left\{C(\delta)|h| /(1+|\lambda|)\left(1+\left|R_{e} \lambda\right|\right)\right\}|f|_{2 \delta}
\end{aligned}
$$

where ||$_{\delta}$ denote the norm of $Q(\delta)$, i.e. $|f|_{s}^{2}=\int_{E^{3}}\left|e^{\delta|x|} f\right|^{2} d x$ and $C(\delta)$ are constants.

2) The case when $b(x) \equiv 0, c(x) \not \equiv 0$.

Lemma 2. Let

$$
L_{1}(\lambda) u=\left(-\Delta+\lambda^{2}+c(x) u\right), u \in \mathscr{D}_{L^{2}}^{2}
$$

and $G_{1}(\lambda)$ be the Green operators of $L_{1}(\lambda)$, that is,

$$
G_{1}(\lambda) \cdot L_{1}(\lambda) \subset L_{1}(\lambda) \cdot G_{1}(\lambda)=I: L^{2} \rightarrow L^{2} .
$$


Then we can consider $G_{1}(\lambda)$ as bounded operators from $Q(\delta)$ to $Q(-\delta)$. In this sense we can analytically continue $G_{1}(\lambda)$ to an analytic function of $\lambda$ in $\operatorname{Re} \lambda \geq-\delta^{\prime}<0$, which satisfies the following estimates (we denote the extension also by $G_{1}(\lambda)$ ),

$$
\begin{aligned}
& \text { i) }\left|G_{1}(\lambda) f\right|_{-\delta} \leq \frac{C}{(1+|\lambda|)(1+|\operatorname{Re} \lambda|)} \mid f !_{\delta} \\
& \text { ii) } \quad\left|\left\{G_{1}(\lambda)-G_{1}(\lambda+h)\right\} f\right|_{-\delta} \leq \frac{C|h|}{(1+|\lambda|)(1+|\operatorname{Re} \lambda|)}|f|_{\delta}
\end{aligned}
$$

and $G_{1}(\lambda)$ are compact operators from $Q(\delta)$ to $Q(-\delta)$ (which map any bounded set to a precompact set), where $c(x) \geq 0$ is a bounded function with compact support.

3) The case when $b(x) \not \equiv 0$.

Lemma 3. Let

$$
L_{2}(\lambda) u=\left(-\Delta+\lambda^{2}+c(x)+\lambda b(x)\right) u, \quad u \in \mathscr{D}_{L^{2}}^{2},
$$

and $G_{2}(\lambda)$ be the Green operators of $L_{2}(\lambda)$, that is,

$$
G_{2}(\lambda) \cdot L_{2}(\lambda) \subset L_{2}(\lambda) \cdot G_{2}(\lambda)=I: L^{2} \rightarrow L^{2} .
$$

Then we can consider $G_{2}(\lambda)$ as bounded operators from $Q(\delta)$ to $Q(-\delta)$. In this sense we can analytically continue $G_{2}(\lambda)$ to analytic function of $\lambda$ in $\operatorname{Re} \lambda \leq-\delta^{\prime \prime}<0$, which satisfies the following estimate (we denote the extension also by $G_{2}(\lambda)$ ),

$$
\left|G_{2}(\lambda) f\right|_{-\delta} \leq \frac{C}{(1+|\lambda|)(1+|\operatorname{Re} \lambda|)}|f|_{\delta} .
$$

Where $b(x) \geq 0$ and $c(x) \geq 0$ are bounded functions with compact supports.

\section{§3. Proof of lemmas}

1) Proof of Lemma 1 (the case when $c(x) \equiv b(x) \equiv 0)$.

We first prove that $R(\lambda)$ is an analytic function of $\lambda$ which values the bounded operators from $Q(2 \delta)$ to $Q(-2 \delta)$. In order to do so it is sufficient to show that $f(x) \rightarrow \varphi_{i}(x) ; \varphi_{i}(x)=\int_{E^{3}}|x-y|^{i} e^{-\lambda|x-y|} f(y) d y$ $(i=-1,0,1,2 \cdots)$ are bounded operators from $Q(2 \delta)$ to $Q(-2 \delta)$. 
Since $\left|e^{-\lambda|x-y|}\right|=e^{-\operatorname{Re} \lambda^{\prime} x-y \mid} \leq e^{+\delta|x-y|} \leq e^{+\delta^{\prime} x \mid} e^{+\delta|y|}, \operatorname{Re} \lambda \geq-\delta<0$,

$$
\begin{gathered}
\int\left|e^{-2 \delta|x|} \varphi_{i}(x)\right|^{2} d x \leq \int\left\{e^{-2 \varepsilon^{\prime} x^{\prime}} \int|x-y|^{i} e^{-\lambda|x-y|} f(x) d y\right\}^{2} d x \\
\leq \iint\left\{e^{-\delta \mid x^{\prime}}|x-y|^{i} e^{-\delta^{\prime} y^{\prime}}\right\}^{2} d x d y \cdot \int\left|e^{+2 \delta^{\prime} y \mid} f(y)\right|^{2} d y
\end{gathered}
$$

that is,

$$
\left|\varphi_{i}\right|_{-2 \delta} \leq C_{i, \delta}|f|_{2 \delta}
$$

Hence $f(x) \rightarrow \varphi_{i}(x)$ are bounded operators from $Q(+2 \delta)$ to $Q(-2 \delta)$. Next we estimate $R(\lambda)$. Since $Q(\delta)$ densely contains $\mathscr{D}$ (compact support, $C^{\infty}$-function), it is sufficient to show the inequality in the case $f \in \mathscr{D}$. When $\operatorname{Re} \lambda>0$, we can write

$$
\begin{aligned}
R(\lambda) f & =\overline{\mathscr{F}}\left[\frac{1}{(2 \pi|\xi|)^{2}+\lambda^{2}} \mathscr{F}(f)\right] \\
& =\int \frac{e^{2 \pi i x \cdot \xi}}{4 \pi^{2}|\xi|^{2}+\lambda^{2}} \hat{f}(\xi) d \xi, \quad \hat{f}(\xi)=\mathscr{F}(f) \\
& =\sum_{(i, j, k)} \int_{\Gamma(i, j, k)} \frac{e^{2 \pi i x \cdot \xi}}{4 \pi^{2}\left(\xi_{1}^{2}+\xi_{2}^{2}+\xi_{3}^{2}\right)+\lambda^{2}} \hat{f}(\xi) d \xi,
\end{aligned}
$$

where $\mathscr{F}$ and $\overline{\mathscr{F}}$ are Fourier transform and Fourier inverse transform, respectively, $i, j, k$ take a sign of + or - , and

$$
\begin{aligned}
& \Gamma(+,+,+)=[0, \infty) \times[0, \infty) \times[0, \infty) \\
& \Gamma(+,+,-)=[0, \infty) \times[0, \infty) \times(-\infty, 0]
\end{aligned}
$$

Now we prove the estimate $\mathrm{i})$. To do so we divide $\lambda$ into four cases which are $\{\lambda ; \operatorname{Re} \lambda \geq N>0\},\{\lambda ;-\delta \leq \operatorname{Re} \lambda \leq N, \operatorname{Im} \lambda \geq N\},\{\lambda ;-\delta$ $\leq \operatorname{Re} \lambda \leq N, \operatorname{Im} \lambda \leq-N\}$ and $\{\lambda ;-\delta \leq \operatorname{Re} \lambda \leq N,|\operatorname{Im} \lambda| \leq N\}$. When $\lambda$ is in $\{\operatorname{Re} \lambda \geq N>0\}$, it follows that

$$
\sup _{\xi \equiv R^{3}}\left|\frac{1}{(2 \pi|\xi|)^{2}+\lambda^{2}}\right| \leq \frac{C}{(1+|\lambda|)(1+|\operatorname{Re} \lambda|)} .
$$

Hence, we have from (3.1)

$$
\|R(\lambda) f\|_{L^{2}}^{2} \leq \frac{C}{(1+|\lambda|)^{2}(1+|\operatorname{Re} \lambda|)^{2}}\|f\|_{L^{2}}^{2} .
$$

Since $Q(2 \delta) \subset L^{2} \subset Q(-2 \delta),(\delta>0)$, we conclude that 


$$
|R(\lambda) f|_{-2 \delta} \leq \frac{C}{(1+|\lambda|)(1+|\operatorname{Re} \lambda|)}|f|_{2 \delta} .
$$

When $\lambda$ is in $\{-\delta<\operatorname{Re} \lambda \leq N, \operatorname{Im} \lambda \geq N\}$, we consider that $\xi$ is of three dimension complex space: $\boldsymbol{C}^{3}$. Then $\hat{f}(\xi)$ is an analytic function in $C^{3}$ and satisfies that

$$
\sup _{|\mathrm{Im} \xi| \leq A}(1+|\xi|)^{M}|\hat{f(\xi)}| \leq C_{M, A},
$$

where $A$ and $M$ are real numbers and $C_{M, A}$ is a constant depending on $A, M$ and $f$. And $1 / 4 \pi^{2}\left(\xi_{1}^{2}+\xi_{2}^{2}+\xi_{3}^{2}\right)+\lambda^{2}$ is analytic in $\xi$ unless the points are such that $\xi_{1}^{2}+\xi_{2}^{2}+\xi_{3}^{2}=-\lambda^{2} / 4 \pi^{2}$. When $\lambda=\alpha+i \beta, \alpha>0$, $\beta \geq N>0$, we set

$$
R_{(i, j, k)}(\lambda) f \equiv \int_{r(i, j, k)} \frac{e^{2 \pi i x \cdot \xi}}{4 \pi^{2}\left(\xi_{1}^{2}+\xi_{2}^{2}+\xi_{3}^{2}\right)+\lambda^{2}} \hat{f}(\xi) \cdot d \xi .
$$

We move lines of integration of the right hand side of (3.4) as following,

$$
\begin{aligned}
& {[0, \infty) \rightarrow[0,+\delta i / 2 \pi]+[+\delta i / 2 \pi,+\infty+\delta i / 2 \pi) \equiv I_{+}+J_{+}} \\
& (-\infty, 0] \rightarrow[0,-\delta i / 2 \pi]+[-\delta i / 2 \pi,-\infty-\delta i / 2 \pi) \equiv I_{-}+J_{-} .
\end{aligned}
$$

Then

$$
R_{(i, j, k)}(\lambda) f=\int_{\left(I_{i}+J_{i}\right) \times\left(I_{j}+J_{J}\right) \times\left(I_{k}+J_{k}\right)} \frac{e^{2 \pi i x \cdot \xi}}{4 \pi^{2}\left(\xi_{1}^{2}+\xi_{2}^{2}+\xi_{3}^{2}\right)+\lambda^{2}} \hat{f}(\xi) d \xi .
$$

In fact, $e^{2 \pi i x \cdot \xi}\left\{4 \pi^{2}\left(\xi_{1}^{2}+\xi_{2}^{2}+\xi_{3}^{2}\right)+\lambda^{2}\right\}^{-1}$ is analytic and bounded in $\xi$ on $D_{i} \times D_{j} \times D_{k}$, and (3.3) holds there, where

$$
D_{+}=\{\operatorname{Re} \eta \geq 0, \delta / 2 \pi \geq \operatorname{Im} \eta \geq 0\}, D_{-}=\{\operatorname{Re} \eta \leq 0,-\delta / 2 \pi \leq \operatorname{Im} \eta \geq 0\} .
$$

(3.5) shows that $R_{(i, j, k)}(\lambda) f$ is analytic in $\{\operatorname{Re} \lambda>-\delta, \operatorname{Im} \lambda \geq N\}$ as a $Q(-\delta)$-valued function of $\lambda$. Let

$$
S\left(K_{i}, K_{j}, K_{k}\right)(\lambda) f \equiv \int_{K_{i} \times K_{3} \times K_{k}} \frac{e^{2 \pi i x \cdot \xi}}{4 \pi^{2}\left(\xi_{1}^{2}+\xi_{2}^{2}+\xi_{3}^{2}\right)+\lambda^{2}} \hat{f}(\xi) d \xi
$$

where $K_{i}$ is $I_{i}$ or $J_{i}$.

Lemma 4. Let $p(\xi, \lambda)=1 /\left|4 \pi^{2}\left(\xi_{1}^{2}+\xi_{2}^{2}+\xi_{3}^{2}\right)+\lambda^{2}\right|$ and $\lambda \in\{-\delta<\operatorname{Re} \lambda$ $\leq N, \operatorname{Im} \lambda \geq N\}$. Then 

i) $\sup _{\xi \equiv I_{1} \times I, \times I_{k}} p(\xi, \lambda) \leq \frac{C}{|\lambda|^{2}}$
ii) $\sup _{\xi=K_{i} \times K_{j} \times K_{k}} p(\xi, \lambda) \leq \frac{C}{(1+|\lambda|)(\delta+\operatorname{Re} \lambda)}$

where $K_{k}$ is $I_{h}$ or $J_{h}$ and one of $K_{i}, K_{j}$ and $K_{k}$ is $J_{+}$or $J_{-}$.

Proof. We may assume $N \geq \delta$. Then $\operatorname{Re} \lambda^{2} \leq 0$.

i) Since $4 \pi^{2}\left(\xi_{1}^{2}+\xi_{2}^{2}+\xi_{3}^{2}\right)=z \in\left[-3 \delta^{2}, 0\right]$ if $\xi \in I_{i} \times I_{j} \times I_{k}$, we have from $\operatorname{Re} \lambda^{2} \leq 0$

$$
p(\xi, \lambda) \leq \sup _{z \equiv\left[-3 \delta^{2}, 0\right]}\left(\frac{1}{\left|\lambda^{2}+z\right|}\right) \leq \frac{C}{|\lambda|^{2}} .
$$

ii) Since $\xi \in K_{i} \times K_{j} \times K_{k}$ and one of $K_{i}, K_{j}$ and $K_{k}$ is $J_{+}$or $J_{-}$, we can write

$$
4 \pi^{2}\left(\xi_{1}^{2}+\xi_{2}^{2}+\xi_{3}^{2}\right)=4 \pi^{2} z^{2} t(\xi), \quad z \in J_{+}, t(\xi) \geq 1 .
$$

Hence

$$
\begin{aligned}
\sup _{\xi \equiv X_{1} \times K_{j} \times K_{k}} p(\xi, \lambda) & \leq \sup _{Z \equiv J_{+}} \frac{1}{\left|\lambda^{2}+4 \pi^{2} z^{2}\right|} \\
& \leq \frac{C}{(|\lambda|+1)(\operatorname{Re} \lambda+\delta)} .
\end{aligned}
$$

This proves Lemma 4.

$1^{\circ} \quad S\left(I_{+}, I_{+}, I_{+}\right)(\lambda) f \equiv \int_{I_{+} \times I_{+} \times I_{+}} \frac{e^{2 \pi i x \cdot \xi}}{4 \pi^{2}\left(\xi_{1}^{2}+\xi_{2}^{2}+\xi_{3}^{2}\right)+\lambda^{2}} \hat{f}(\xi) d \xi$

$$
=-i \int_{0}^{\delta / 2 \pi} \int_{0}^{\delta / 2 \pi} \int_{0}^{\delta / 2 \pi} \frac{e^{-2 \pi\left(s_{1} x_{1}+s_{2} x_{2}+s_{3} x_{2}\right)}}{\lambda^{2}-4 \pi^{2}\left(s_{1}^{2}+s_{2}^{2}+s_{3}^{2}\right)} \hat{f}(+i s) d s_{1} d s_{2} d s_{3}, s=\left(s_{1}, s_{2}, s_{3}\right) \text {. }
$$

Since

$$
\begin{aligned}
& \mid\left. f(\text { is })\right|^{2} \leq\left\{\int\left|e^{2 \pi\left(s_{1} x_{1}+s_{2} x_{2}+s_{3} x_{3}\right)}\right| f|(x)| d x\right\}^{2} \\
& \quad \leq \int e^{-2 \varepsilon|x|} d s \cdot \int\left|e^{(\sqrt{3} \bar{\delta}+\varepsilon)|x|} f(x)\right|^{2} d x, \quad\left(\left|s_{1}\right|,\left|s_{2}\right|,\left|s_{3}\right| \leq \frac{\delta}{2 \pi}\right), \\
& \int\left|e^{-(\sqrt{3} \delta+\varepsilon) \mid x} S\left(I_{+}, I_{+}, I_{+}\right)(\lambda) f\right|^{2} d x \\
& \quad \leq\left\{\int e^{-2 \varepsilon|x|} d x\right\}^{2}\left\{\iiint_{0}^{2 \pi} d s_{1} d s_{2} d s_{3}\right\}^{2} \cdot \int\left|e^{(\sqrt{3} \delta+\varepsilon)^{\prime} x \mid} f(x)\right|^{2} d x \\
& \quad \times \sup _{0 \leq s, s, s \leq \delta / 2 \pi}\left|\frac{1}{\lambda^{2}-4 \pi^{2}\left(s_{1}^{2}+s_{2}^{2}+s_{3}^{2}\right)}\right| .
\end{aligned}
$$

From Lemma 4, i) 


$$
\leq \frac{(4 \pi)^{2}}{(2 \varepsilon)^{6}}\left(\frac{\delta}{2 \pi}\right)^{6} \int\left|e^{(\sqrt{3} \delta+\varepsilon) x^{\prime}} f(x)\right|^{2} d x \cdot \frac{C}{|\lambda|^{4}} .
$$

$2^{\circ} \quad S\left(I_{+}, J_{+}, J_{+}\right)(\lambda) f \equiv \int_{I_{+} \times J_{+} \times J_{+}} \frac{e^{2 \pi i x \cdot \xi}}{4 \pi^{2}\left(\xi_{1}^{2}+\xi_{2}^{2}+\xi_{3}^{2}\right)+\lambda^{2}} \hat{f}(\xi) d \xi$

$$
=i \int_{0}^{2 \pi} d s_{1} \int_{0}^{\infty} d s_{2} \int_{0}^{\infty} \frac{e^{-2 \pi s_{1} x_{1}} e^{2 \pi i s_{2} x_{2}-\delta x_{2}} e^{2 \pi i s_{3 z_{3}-\delta x_{3}}}}{4 \pi^{2}\left\{\left(i s_{1}\right)^{2}+\left(s_{2}+i \frac{\delta}{2 \pi}\right)^{2}+\left(s_{3}+i \frac{\delta}{2 \pi}\right)^{2}\right\}+\lambda^{2}} \hat{f}(\sigma) d s_{3},
$$

where $\sigma$ denotes $\left(i s_{1}, s_{2}+i \frac{\delta}{2 \pi}, s_{3}+i \frac{\delta}{2 \pi}\right)=i e^{-\delta x_{2}-\delta x_{3}}$

$$
\times \int_{0}^{\infty} \int_{0}^{\infty} e^{2 \pi i\left(s_{2} x_{2}+s_{3} x_{3}\right)}\left\{\int_{0}^{S / 2 \pi} \frac{e^{-2 \pi s_{1} x_{1}} g_{1}\left(s_{1}, s_{2}, s_{3}\right)}{q_{1}\left(s_{1}, s_{2}, s_{3}, \lambda\right)} d s_{1}\right\} d s_{2} d s_{3}
$$

where

$$
\left\{\begin{array}{l}
q_{1}\left(s_{1}, s_{2}, s_{3}, \lambda\right)=4 \pi^{2}\left\{\left(s_{2}+i \frac{\delta}{2 \pi}\right)^{2}+\left(s_{3}+i \frac{\delta}{2 \pi}\right)^{2}-s_{1}^{2}\right\}+\lambda^{2} \\
g_{1}\left(s_{1}, s_{2}, s_{3}\right)=\hat{f}\left(i s_{1}, s_{2}+i \frac{\delta}{2 \pi}, s_{3}+i \frac{\delta}{2 \pi}\right)
\end{array}\right.
$$

Since we can regard the above equality as Fourier transform from $\left(s_{2}, s_{3}\right)$ to $\left(x_{2}, x_{3}\right)$, using Plancherel's theorem, we have the following inequality.

$$
\begin{aligned}
& \iint\left|e^{\delta x_{2}+\delta x_{3}} S\left(I_{+}, J_{+}, J_{+}\right)(\lambda) f\right|^{2} d s_{2} d s_{3} \\
= & \iint\left|\int_{0}^{\delta / 2 \pi} \frac{e^{-\pi s_{1} x_{1}}}{q_{1}\left(s_{1}, s_{1}, s_{2}, s_{3}\right)} d s_{1}\right|^{2} d s_{2} d s_{3} \\
& \leq \underset{\inf _{\left(s_{1}, s_{2}, s_{3}\right)}\left|q_{1}\left(s_{1}, s_{2}, s_{3}\right)\right|}{q^{2 \delta \mid x_{1} !}}\left(\frac{\delta}{2 \pi}\right) \iint_{0}^{-\infty}\left\{\int_{0}^{-2 / 2 \pi}\left|g_{1}\left(s_{1}, s_{2}, s_{3}\right)\right|^{2} d s_{1}\right\} d s_{2} d s_{3}
\end{aligned}
$$

and

$$
\iint_{0}^{\infty}\left|g_{1}\left(s_{1}, s_{2}, s_{3}\right)\right|^{2} d s_{2} d s_{3} \leq \iint_{-\infty}^{+\infty}\left|\hat{f}\left(i s_{1}, s_{2}+i \frac{\delta}{2 \pi}, s_{3}+i \frac{\delta}{2 \pi}\right)\right|^{2} d s_{2} d s_{3}
$$

also using Plancherel's theorem on $\left(y_{2}, y_{3}\right) \rightarrow\left(s_{2}, s_{3}\right)$,

$$
\begin{aligned}
& =\iint_{-\infty}^{+\infty}\left|\int_{-\infty}^{+\infty} e^{\delta y_{3}+\delta y_{3}} e^{2 \pi s_{1} y_{1}} f(y) d y_{1}\right|^{2} d y_{2} d y_{3} \\
& \quad \leq \int_{-\infty}^{+\infty} e^{-2 \varepsilon\left|y_{1}\right|} d y_{1} \cdot \iiint\left|e^{\sqrt{3} \delta|y|+\varepsilon\left|y_{1}\right|} f(y)\right|^{2} d y_{1} d y_{2} d y_{3} .
\end{aligned}
$$

In fact, $\left|\delta y_{1}+\delta y_{2}+\delta y_{3}\right| \leq \sqrt{3} \delta|y|$.

Since 


$$
\begin{aligned}
\inf _{\substack{\left(0 \leq s_{1} \leq \delta / 2 \pi\right) \\
\left(0 \leq s_{2}, s_{3}<\infty\right)}}\left|q_{1}\left(s_{1}, s_{2}, s_{3}, \lambda\right)\right|^{2} & =\left\{\sup _{\xi \equiv I_{+} \times J_{+} \times J_{+}} p(\xi, \lambda)^{2}\right\}^{-1} \\
& \geq C(1+|\lambda|)^{2}(\delta+\operatorname{Re} \lambda)^{2}
\end{aligned}
$$

from Lemma 4, ii), it follows that

$$
\begin{aligned}
& \int\left|e^{-(\sqrt{3} \delta+\varepsilon)|x|} S\left(I_{+}, J_{+}, J_{+}\right)(\lambda) f\right|^{2} d x \\
& \quad \leq \int_{-\infty}^{\infty} e^{-2(\delta+\varepsilon) x_{1} \mid}\left\{\iint_{-\infty}^{+\infty}\left|e^{\delta x_{2}{ }^{\perp} \delta x_{3}} \cdot S\left(I_{+}, J_{+}, J_{+}\right)(\lambda) f\right|^{2} d x_{2} d x_{3}\right\} d x_{1} \\
& \quad \leq C(1+|\lambda|)^{-2}(\delta+\operatorname{Re} \lambda)^{-2} \cdot\left\{\int_{-\infty}^{+\infty} e^{-2 \varepsilon\left|x_{1}\right|} d x_{1}\right\}^{2}\left(\frac{\delta}{2 \pi}\right)^{2} \\
& \quad \times \int_{R^{3}}\left|e^{\left(\sqrt{3} \overline{3}^{+} \varepsilon\right)|x|} f(x)\right|^{2} d x .
\end{aligned}
$$

$3^{\circ} \quad S\left(J_{+}, J_{+}, J_{+}\right)(\lambda) f \equiv \int_{J_{+} \times J_{+} \times J_{+}} \frac{e^{2 \pi i x \xi}}{4 \pi^{2}\left(\xi_{1}^{2}+\xi_{2}^{2}+\xi_{3}^{2}\right)+\lambda^{2}} \hat{f}(\xi) d \xi$

$$
=e^{-\delta\left(x_{1}+x_{2}+x_{3}\right)} \int_{0}^{\infty} \int_{0}^{\infty} \int_{0}^{\infty} e^{2 \pi i\left(x_{1} s_{1}+x_{2} s_{2}+x_{3} s_{3}\right)} \frac{g_{2}\left(s_{1}, s_{2}, s_{3}\right)}{q_{2}\left(s_{1}, s_{2}, s_{3}, \lambda\right)} d s_{1} d s_{2} d s_{3}
$$

where

$$
\left\{\begin{array}{l}
g_{2}\left(s_{1}, s_{2}, s_{3}\right)=\hat{f}\left(s_{1}+i \frac{\delta}{2 \pi}, s_{2}+i \frac{\delta}{2 \pi}, s_{3}+i \frac{\delta}{2 \pi}\right) \\
q_{2}\left(s_{1}, s_{2}, s_{3}, \lambda\right)=4 \pi^{2}\left\{\left(s_{1}+i \frac{\delta}{2 \pi}\right)^{2}+\left(s_{2}+i \frac{\delta}{2 \pi}\right)^{2}+\left(s_{3}+i \frac{\delta}{2 \pi}\right)^{2}\right\}+\lambda^{2}
\end{array}\right.
$$

In the same manner with $2^{\circ}$, using Plancherel's theorem and Lemma 4, ii), we have

$$
\begin{aligned}
\int_{R^{3}}\left|e^{-(\sqrt{3} 3 \delta+\varepsilon)|x|} S\left(J_{+}, J_{+}, J_{+}\right)(\lambda) f\right|^{2} d x \\
\quad \leq\left.\int_{R^{3}} e^{\delta\left(x_{1}+x_{2}+x_{3}\right)} S\left(J_{+}, J_{+}, J_{+}\right)(\lambda) f\right|^{2} d x \\
\leq C(1+|\lambda|)^{-2}(\delta+\operatorname{Re} \lambda)^{-2} \int_{R^{3}}\left|e^{-\delta\left(x_{1}+x_{2}+x_{3}\right)} f(x)\right|^{2} d x \\
\leq C(1+|\lambda|)^{-2}(\delta+\operatorname{Re} \lambda)^{-2} \int_{R^{3}}\left|e^{(\sqrt{3} \bar{\delta}+\varepsilon)|x|} f(x)\right|^{2} d x
\end{aligned}
$$

In the same manner we have the estimates of other $S(\cdots)(\lambda) f$, that is,

$$
\begin{aligned}
& \int\left|e^{-(\sqrt{3}+\varepsilon)|x|} S(\cdots)(\lambda) f\right|^{2} d x \\
& \quad \leq C(1+|\lambda|)^{-2}(\delta+\operatorname{Re}|\lambda|)^{-2} \int\left|e^{(\sqrt{3} \delta+\varepsilon)|x|} f\right|^{2} d x .
\end{aligned}
$$


Thus, from (3.2), (3.4), (3.6) and (3.7) we can estimate $R(\lambda) f$ as following.

$$
\begin{aligned}
R(\lambda) f & =\sum_{(1, j, k)} R_{(i, j, k)}(\lambda) f \\
& =\sum_{(i, j, k)}\left\{\sum_{\left(K_{i}, K_{j}, K_{k}\right)} S\left(K_{i}, K_{j}, K_{k}\right)(\lambda) f\right\} .
\end{aligned}
$$

Hence

$$
\begin{aligned}
& \int_{R^{3}}\left|e^{-\gamma|x|} R(\lambda) f\right|^{2} d x \\
& \quad \leq \sum_{(i, j, k)}\left\{\sum_{\left(K_{i}, K_{j}, K_{k}\right)} \int_{R^{3}}\left|e^{-\gamma^{\prime} x \mid} S\left(K_{i}, K_{j}, K_{k}\right)(\lambda) f\right|^{2} d x\right\} \\
& \leq C(1+|\lambda|)^{-2}(\delta+\operatorname{Re} \lambda)^{-2} \int_{R^{3}}\left|e^{\gamma|x|} f(x)\right|^{2} d x .
\end{aligned}
$$

Where $\lambda=\alpha+i \beta,-\delta<\alpha \leq N, \beta \geq N$ and $\gamma=\sqrt{3} \delta+\varepsilon>0$. If we take $\varepsilon$ such that $\gamma \leq 2 \delta$, we have the estimate of Lemma 1 .

When $\operatorname{Im} \lambda \leq-N<0$, we can have the estimates in the same manner with the above case if we move lines of integration of the right hand side of (3.4) as following

$$
\begin{aligned}
& {[0, \infty) \rightarrow\left[0,-i \frac{\delta}{2 \pi}\right]+\left[-i \frac{\delta}{2 \pi},+\infty-i \frac{\delta}{2 \pi}\right)} \\
& (-\infty, 0] \rightarrow\left[0,+i \frac{\delta}{2 \pi}\right]+\left[+i \frac{\delta}{2 \pi},-\infty+i \frac{\delta}{2 \pi}\right) .
\end{aligned}
$$

Thus we obtain the estimate i) of Lemma 1 . We can prove the estimates ii), iii) and iv) of Lemma 1 , using the fact that $p_{1}(\lambda), p_{2}(\lambda)$ and $p_{3}(\lambda)$ satisfy the following inequalities,

$$
\begin{aligned}
& \sup _{\xi \Subset K_{i} \times K_{j} \times K_{k}}\left|p_{1}(\lambda)\right|^{2} \leq C(\delta+\operatorname{Re} \lambda)^{-2} \\
& \sup _{\xi \in K_{\imath} \times K_{j} \times K_{k}}\left|p_{2}(\lambda)\right|^{2} \leq C(1+|\lambda|)^{2}(\delta+\operatorname{Re} \lambda)^{-2} \\
& \sup _{\xi \Subset K_{1} \times K_{j} \times K_{k}}\left|p_{3}(\lambda)\right|^{2} \leq C \frac{|h|^{2}|2 \lambda+h|^{2}}{(1+|\lambda|)^{4}(\delta+\operatorname{Re} \lambda)^{2}(\delta+\operatorname{Re} \lambda+h)^{2}}
\end{aligned}
$$

where

$$
\begin{aligned}
& p_{0}(\lambda)=\left\{4 \pi^{2}\left(\xi_{1}^{2}+\xi_{2}^{2}+\xi_{3}^{2}\right)+\lambda^{2}\right\}^{-1} \\
& p_{1}(\lambda)=2 \pi \xi_{\nu} p_{0}(\lambda), \quad p_{2}(\lambda)=4 \pi^{2} \xi_{\nu} \cdot \xi_{\mu} p_{0}(\lambda) \\
& p_{3}(\lambda)=p_{0}(\lambda+h)-p_{0}(\lambda)=-p_{0}(\lambda+h) p_{0}(\lambda) h(2 \lambda+h) .
\end{aligned}
$$

The proof of Lemma 1 is complete. 
2) Proof of Lemma 2 (the case when $b(x) \equiv 0, c(x) \equiv 0$ ).

Lemma 5. Let $c(x)$ be a bounded function with compact support and $c(x) \geq 0$. Let $u(x)$ satisfy that

$$
u(x)+\frac{1}{4 \pi} \int_{E^{3}} \frac{e^{-\lambda^{\prime} x-y \mid}}{|x-y|} c(y) u(y) d y=0, \quad u \in L_{l o c}^{2} .
$$

Then $u(x) \equiv 0$ in $E^{3}$ if $\operatorname{Re} \lambda \geq 0$.

Proof. From (3.8) and the assumption that $c(x)$ is a bounded function with compact support, we have the condition of $u(x)$ such that

$$
\begin{gathered}
\left\{-\Delta+\lambda^{2}+c(x)\right\} u(x)=0 \\
u(x)=0\left(|x|^{-1}\right) e^{-\operatorname{Re} \lambda|x|}, \frac{d}{d x} u(x)+\lambda u(x)=0\left(|x|^{-1}\right) e^{-R e \lambda|x|}
\end{gathered}
$$

as $|x| \rightarrow \infty$. Let $\lambda=\alpha+i \beta$. When $\beta=0$, we have from (3.9) that

$$
\begin{aligned}
0= & \int_{|x| \leq R}\left\{-\Delta+c(x)+\alpha^{2}\right\} u(x) \cdot \overline{u(x)} d x \\
= & \int_{|x| \leq R}|\operatorname{grad} u(x)|^{2} d x+\int_{|x| \leq R} c(x)|u(x)|^{2} d x \\
& \quad+\alpha^{2} \int_{|x| \leq R}|u(x)|^{2} d x-\int_{|x|=R}\left(\frac{d}{d|x|} u\right) \bar{u} d s .
\end{aligned}
$$

Since the last term of (3.11) vaishes when $R \rightarrow \infty$ and $c(x) \geq 0$, we have that

$$
\int_{|x| \leq R}|\operatorname{grad} u(x)|^{2} d x=0 \text {, that is, }|\operatorname{grad} u(x)|=0 .
$$

This implies that $u(x)$ is constant. Thus $u(x) \equiv 0$ in $E^{3}$ from (3.10) if $\alpha \geq 0$. When $\alpha \beta \neq 0$, we have $u(x) \equiv 0$ since $2 \alpha \beta \int|u(x)|^{2} d x=0$ which is the imaginary part of $\int\left\{-\Delta+\lambda^{2}+c(x)\right\} u(x) \cdot \overline{u(x)} d x$. The case when $\alpha=0$ and $\beta \neq 0$ is left. In this case it is sufficient to prove the two fact such that a) $u(x)$, which satisfies (3.8), is a function of $L^{2}$; b) if $\left(\Delta+\beta^{2}\right) u(x)=0$ at $|x| \geq R$ and $u(x) \in L^{2}(|x| \geq R)$, then $u(x) \equiv 0$ in $|x| \geq R$. In fact, if $u(x) \equiv 0$ in $|x| \geq R, u(x) \equiv 0$ in whole space $E^{3}$ from the unique continuation theorem of solutions of elliptic equation of second order (Refer to Eìdus [3] or Povzner [4] for details of Lemma 5). q. e. d. 
We now prove Lemma 2. Let $\mathcal{A}(\delta)$ be the Banach space of bounded operators from $Q(-\delta)$ to $Q(-\delta)$ and let $\mathcal{B}(\delta)$ be that from $Q(\delta)$ to $Q(-\delta)$. We denote the norm of each Banach space by

$$
\begin{aligned}
|A|_{\delta} \equiv \sup _{\mid f-\delta \leq 1}|A f|_{-\delta}, & A \in \mathcal{A}(\delta) \\
|B|_{\delta} \equiv \sup _{\mid f_{\delta} \leq 1}|B f|_{-\delta}, & B \in \mathscr{B}(\delta) .
\end{aligned}
$$

Let $u(x, \lambda)$ be a solution in $L^{2}$ of the equation $L_{1}(\lambda) u=f$ where $f \in L^{2}$ and $\operatorname{Re} \lambda>0$. Then $u(x, \lambda)$ is a solution of the integral equation

$$
u(x, \lambda)+R(\lambda) \cdot c(x) \cdot u(x, \lambda)=R(\lambda) \cdot f(x)
$$

which is obtained by operating $R(\lambda)$ to the both sides of $L_{1}(\lambda) u=f$, where $R(\lambda)$ is defined in Lemma 1 and $c(x) \cdot$ is an operator which multiplies $c(x)$. That is,

$$
\{I+R(\lambda) \cdot c(x) \cdot\} G_{1}(\lambda)=R(\lambda), \quad \text { at } \operatorname{Re} \lambda>0,
$$

where $I+R(\lambda) \cdot c(x), G_{1}(\lambda)$ and $R(\lambda)$ are bounded operators on $L^{2}$. To obtain the analytic continuation of $G_{1}(\lambda)$ we shall show that the equation of (3.12) can be solved at $\operatorname{Re} \lambda \leq 0$ if we consider $G_{1}(\lambda)$ as an element of $\mathscr{B}(\boldsymbol{\delta})$.

(3.13): $R(\lambda) \in \mathscr{B}(\delta)$ and $R(\lambda) \cdot c(x) \cdot \in \mathcal{A}(\delta)$ are compact operators, that is, they map a bounded set to a pre-compact set, and they are analytic functions of $\lambda$, which value in $\mathcal{B}(\delta)$ and $\mathcal{A}(\delta)$, respectively, at $\operatorname{Re} \lambda \geq-\delta^{\prime}$ for some $\delta^{\prime}>0$.

In fact an integral operator having a kernel $e^{-\delta|x|} \frac{e^{-\lambda|x-y|}}{|x-y|} e^{-\delta|y|}$, which is the Hilbert-Schmidt type, is a compact operator on $L^{2}, e^{+\delta|x|}$ is a bounded operator from $Q(\gamma)$ to $Q(\gamma-\delta)$, and $c(x)$. is a bounded operator from $Q(-\delta)$ to $Q(\delta)$ from the assumption that $c(x)$ is a bounded function with compact support. Hence we have that $R(\lambda)$ and $R(\lambda) \cdot c(x) \cdot$ are compact operators. The analyticity follows from Lemma 1 and the assumption for $c(x)$.

(3.14): $\{I+R(\lambda) \cdot c(x) \cdot\}$ has an inverse $\{I+R(\lambda) \cdot c(x) \cdot\}^{-1} \in \mathcal{A}(\delta)$ at $\operatorname{Re} \lambda \geq 0$.

In fact, since $R(\lambda) \cdot c(x) \cdot$ is a compact operator on $Q(-\delta)$ from (3.13) 
it is sufficient to show that, if $\{I+R(\lambda) \cdot c(x) \cdot\} u=0$ and $u \in Q(-\delta)$, then $u=0$. This follows from Lemma 5 . Let us put

$$
R_{1}(\lambda)=\{I+R(\lambda) \cdot c(x) \cdot\}^{-1} .
$$

We now assume that there exists the inverse $R_{1}(\lambda)$ at $\lambda=\lambda_{0}$. If we operate $R_{1}\left(\lambda_{0}\right)$ to the both sides of $\{I+R(\lambda) \cdot c(x) \cdot\} u=f ; \quad u$ and $f \in Q(-\delta)$, we have

$$
\{I+S(\lambda)\} u=R_{1}\left(\lambda_{0}\right) f \text {, where } S(\lambda)=R_{1}\left(\lambda_{0}\right)\left\{R(\lambda)-R\left(\lambda_{0}\right)\right\} C(x):
$$

Since $R(\lambda) \cdot c(x) \cdot$ is analytic at $\lambda=\lambda_{0}$, we have $S(\lambda)_{1} \leq 1 / 2$ in some neighborhood of $\lambda=\lambda_{0}$. Hence the Neumann series

$$
\{I+S(\lambda)\}^{-1}=I+S(\lambda)+S(\lambda)^{2}+\cdots
$$

uniformly converge in the neighborhood of $\lambda=\lambda_{0}$. This implies that $\{I+S(\lambda)\}^{-1}$ is analytic in the neighborhood of $\lambda=\lambda_{0}$ for $S(\lambda)$ is analytic there. Thus, there exists $R_{1}(\lambda) \equiv\{I+S(\lambda)\}^{-1} R_{1}\left(\lambda_{0}\right)$ which is analytic in the neighborhood of $\lambda=\lambda_{0}$. Considering (3.14) and above, we have that

(3.15): $R_{1}(\lambda)=\{I+R(\lambda) \cdot c(x) \cdot\}^{-1}$ is an analytic function of $\lambda$ at $\operatorname{Re} \lambda \geq 0$ which values in $\mathcal{A}(\boldsymbol{\delta})$.

Let us estimate $R_{1}(\lambda)$. From Lemma 1 , i) we have, at $\operatorname{Re} \geq-\delta / 2$,

$$
\begin{aligned}
& |R(\lambda) \cdot c(x) \cdot f(x)|_{-\delta} \leq C(1+|\lambda|)^{-1}(1+|\operatorname{Re} \lambda|)^{-1} \cdot|c(x) \cdot f(x)|_{\delta} \\
& \quad \leq C(1+|\lambda|)^{-1}(1+|\operatorname{Re} \lambda|)^{-1} \sup _{x}\left|c(x) \cdot e^{2 \delta|x|}\right| \cdot|f(x)|_{-i},
\end{aligned}
$$

that is,

$$
\left\|_{\|} R(\lambda) \cdot c(x) \cdot\right\|_{\delta} \leq C(1+|\lambda|)^{-1}(1+|\operatorname{Re} \lambda|)^{-1} .
$$

Hence, since $\|R(\lambda) \cdot c(x) \cdot\|_{\delta} \leq 1 / 2$ when $|\lambda|$ is large enough and $\operatorname{Re} \lambda \geq-\delta / 2$, there exists the inverse of $\{I+R(\lambda) \cdot c(x) \cdot\}$ by Neumann's series;

$$
R_{1}(\lambda)=I+R(\lambda) \cdot c(x) \cdot+(R(\lambda) \cdot c(x) \cdot)^{2}+\cdots,
$$

which implies that $R_{1}(\lambda)$ is analytic in $\lambda$ and $\left.{ }_{\|} R_{1}(\lambda)\right|_{10} \leq$ Const. in $\operatorname{Re} \lambda \geq-\delta / 2$ and $|\lambda| \geq M$. Since (3.15) holds where $|\lambda|$ is bounded, we have that 
(3.16): $R_{1}(\lambda)$ can be continued to an analytic function in $\operatorname{Re} \lambda \geq-\delta^{\prime}$, $\delta^{\prime}>0$, which value $\mathcal{A}(\delta)$. If we denote the extension also by $R_{1}(\lambda)$, we can estimate $R_{1}(\lambda)$ as following,

$$
R_{1}(\lambda) ; \delta \leq \text { Const. }<\infty ; \quad \operatorname{Re} \lambda \geq-\delta^{\prime} .
$$

Using (3.16) and Lemma 1, we conclude that

(3.17): $G_{1}(\lambda)$ can be continued to an analytic function in $\operatorname{Re} \lambda \geq-\delta^{\prime}$, $\delta^{\prime}>0$, which value $\mathcal{B}(\delta)$ and has the estimates as

$1^{\circ}\left|G_{1}(\lambda)\right|_{\delta} \leq$ Const. $(1+|\lambda|)^{-1}(1+|\operatorname{Re} \lambda|)^{-1}$

$2^{\circ}\left|G_{1}(\lambda)-G_{1}(\lambda+h)\right|_{\delta} \leq$ Const. $|h|(1+|\lambda|)^{-1}(1+|\operatorname{Re} \lambda|)^{-1}$, $0 \leq \operatorname{Re} h<1, \quad|h| \leq 1$.

In fact, from (3.12) we have $G_{1}(\lambda)=R_{1}(\lambda) \cdot R(\lambda)$ at $\operatorname{Re} \lambda>0$, the right hand side of which is analytic in $\operatorname{Re} \lambda \geq-\delta^{\prime}$ from (3.16). This defines the continuation. Since $\left|G_{1}(\lambda)\right|_{\delta} \leq \|\left. R_{1}(\lambda)\right|_{\delta} \cdot|R(\lambda)|_{\delta}$, the estimate $1^{\circ}$ of (3.17) follows from the estimates of (3.16) for $R_{1}(\lambda)$ and of Lemma 1 , i) for $R(\lambda)$ which is $|R(\lambda)|_{\delta} \leq C(1+|\lambda|)^{-1}(1+|\operatorname{Re} \lambda|)^{-1}$.

Using (3.12), we have

$$
\begin{aligned}
& \{I+R(\lambda) \cdot c(x) \cdot\} \cdot\left\{G_{1}(\lambda)-G_{1}(\lambda+h)\right\} \\
& \quad=\{R(\lambda+h)-R(\lambda)\} \cdot c(x) \cdot G_{1}(\lambda+h)-\{R(\lambda+h)-R(\lambda)\} .
\end{aligned}
$$

Operating $R_{1}(\lambda) \equiv\{I+R(\lambda) \cdot c(x) \cdot\}^{-1}$ to the both sides of above equality,

$$
\begin{aligned}
& \left|G_{1}(\lambda)-G_{1}(\lambda+h)\right|_{\delta} \\
& \leq\left|R_{1}(\lambda)\left[\{R(\lambda+h)-R(\lambda)\} \cdot c(x) \cdot G_{1}(\lambda+h)-\{R(\lambda+h)-R(\lambda)\}\right]\right|_{\delta} \\
& \leq\left.\left|R_{1}(\lambda)\right|_{\delta} \cdot[R(\lambda+h)-R(\lambda)\} \cdot c(x)\right|_{\delta} \cdot\left|G_{1}(\lambda+h)\right|_{\delta} \\
& \left.+|\{R(\lambda+h)-R(\lambda)\}|_{\delta}\right] .
\end{aligned}
$$

Using the estimates of iv) of Lemma 1, of (3.16) and of $1^{\circ}$ of (3.17) and the inequality;

$$
\text { ' }\{R(\lambda+h)-R(\lambda)\} \cdot c(x){ }_{\delta} \leq \text { Const. }|R(\lambda+h)-R(\lambda)|_{\delta},
$$

we have the estimate $2^{\circ}$ of $(3.17)$, that is,

$$
\begin{aligned}
\mid G_{1}(\lambda) & -\left.G_{1}(\lambda+h)\right|_{\delta} \\
& \leq|\{R(\lambda+h)-R(\lambda)\}|_{\delta}\left\{\text { Const. } \| R_{1}(\lambda)||_{\delta} \cdot\left|G_{1}(\lambda+h)\right|_{\delta}+1\right\} \\
& \leq \text { Const. }|h|(1+|\lambda|)^{-1}(1+|\operatorname{Re} \lambda|)^{-1} ; 0 \leq \operatorname{Re} h,|h| \leq 1 .
\end{aligned}
$$


The proposition of (3.17) is nothing but Lemma 2. Since $R(\lambda)$ is a compact operator and $R_{1}(\lambda)$ is a bounded operator, $G_{1}(\lambda)=$ $R_{1}(\lambda) \cdot R(\lambda)$ is also a compact operator.

The proof of Lemma 2 is complete.

3) Proof of Lemma 3 (the case when $b(x) \not \equiv 0)$.

From the assumption of Lemma 3 , that is, $b(x) \geq 0, c(x) \geq 0$ are bounded functiors with compact supports, we may put $b(x)=a^{2}(x)$, where $a(x)$ is a bounded real valued function with compact support, and there exists the Green operator $G_{1}(\lambda)$ of $L_{1}(\lambda)$ which satisfies Lemma 2.

Operating $G_{1}(\lambda)$ to the both sides of the following equality

$$
\begin{aligned}
& L_{2}(\lambda) u \equiv\left\{-\Delta+\lambda^{2}+c(x)+\lambda b(x)\right\} u=f, \\
& u \in D\left(L_{2}(\lambda)\right), \quad f \in L^{2}, \quad \operatorname{Re} \lambda>0
\end{aligned}
$$

we have

$$
\left\{I+\lambda G_{1}(\lambda) \cdot b(x) \cdot\right\} u(x)=G_{1}(\lambda) \cdot f(x) \equiv g(x) .
$$

From Lemma $2 G_{1}(\lambda) \cdot b(x) \cdot$ is a compact operator on $Q(-\delta)$ at $\operatorname{Re} \lambda \geq-\delta^{\prime}<0$ and $g(x) \in Q(-\delta)$ if $f(x) \in Q(\delta)$. In the same manner as in the proof of Lemma 2 we first prove that

(3.19): There exists the inverse $R_{2}(\lambda) \equiv\left\{I+\lambda G_{1}(\lambda) \cdot b(x) \cdot\right\}^{-1} \in \mathcal{A}(\delta)$ in $\operatorname{Re} \lambda \geq-\delta^{\prime \prime}$ for some positive $\delta^{\prime \prime}$ which is analytic there and satisfies that

$$
\| R_{2}(\lambda){ }_{\delta} \leq \text { Const., }
$$

where $A(\delta)$ is the Banach space defined in the proof of Lemma 2 and \|\|$_{s}$ is the norm of $\mathcal{A}(\delta)$.

It is clear from the analyticity of $\lambda G_{1}(\lambda) \cdot b(x) \cdot$ that $R_{2}(\lambda)$ is analytic where $R_{2}(\lambda)$ exists. To prove (3.19) we note that the following two problems are equivalent, which are to solve the equation;

$$
\left\{I+\lambda G_{1}(\lambda) \cdot b(x) \cdot\right\} u(x)=g(x) \text { in } Q(-\delta)
$$

and to solve in $L^{2}$ the equation;

$$
\left\{I+\lambda a(x) \cdot G_{1}(\lambda) \cdot a(x) \cdot\right\} v(x)=a(x) \cdot g(x) \equiv h(x) .
$$


Here the relations of $u(x)$ and $v(x)$ are given by

$$
\left\{\begin{array}{l}
v(x)=a(x) \cdot u(x) \\
u(x)=-\lambda G_{1}(\lambda) \cdot a(x) \cdot v(x)+g(x) .
\end{array}\right.
$$

We put

$$
T_{\lambda} \equiv \lambda a(x) \cdot G_{1}(\lambda) \cdot a(x) .
$$

Since $T_{\lambda}$ is a compact operator on $L^{2}$, there exists the inverse of $\left\{I+T_{\lambda}\right\}$ if we can show the estimate $\|v\|_{L^{2}} \leq C\left\|\left\{I+T_{\lambda}\right\} v\right\|_{L^{2}}$ for all $v \in L^{2}$. We show it when $\alpha>0 ; \lambda=\alpha+i \beta$. The proof of Lemma 5 gives that $L_{1}=-\Delta+c(x), D\left(L_{1}\right)=\mathscr{D}_{L^{2}}^{2}$ is a positive definite selfadjoint operator, that is, there exists the resolution of the identity $E_{\mu}(0 \leq \mu<\infty)$ such that

$$
L_{1} f=\int_{0}^{\infty} \mu d E_{\mu} f, \text { for all } f \in D\left(L_{1}\right)=\mathscr{D}_{L^{2}}^{2} .
$$

Hence we can write

$$
G_{1}(\lambda) f=\int_{0}^{\infty} \frac{1}{\mu+\lambda^{2}} d E_{\mu} f
$$

Therefore

$$
T_{\lambda} v=\lambda a(x) \cdot \int_{0}^{\infty} \frac{1}{\mu+\lambda^{2}} d E_{\mu} a(x) \cdot v
$$

If we denote the inner product of $L^{2}$ by $($,$) ,$

$$
\begin{aligned}
\operatorname{Re}\left(T_{\lambda} v, v\right)= & \operatorname{Re}\left(a(x) \cdot \int_{0}^{\infty} \frac{\lambda}{\mu+\lambda^{2}} d E_{\mu} a(x) \cdot v, v\right) \\
= & \operatorname{Re} \int_{0}^{\infty} \frac{\lambda}{\mu+\lambda^{2}} d\left(E_{\mu} a(x) \cdot v, a(x) \cdot v\right) \\
= & \int_{0}^{\infty} \frac{\left(\mu+a^{2}+\beta^{2}\right) \alpha}{\left(\mu+\alpha^{2}-\beta^{2}\right)^{2}+(2 \alpha \beta)^{2}} d\left(E_{\mu} a(x) \cdot v, a(x) \cdot v\right) ; \\
& \lambda=\alpha+i \beta, \alpha>0 .
\end{aligned}
$$

Since $\left(E_{\iota} a(x) \cdot v, a(x) \cdot v\right)$ is a monotone increasing function and the integrand is a positive function from $a>0$ and $\mu \geq 0$, it follows that

$$
\operatorname{Re}\left(T_{\mu} v, v\right) \geq 0, \quad \operatorname{Re} \lambda>0, \text { for all } v \in L^{2} .
$$

Applying it to the equality;

$$
\left\|v_{L^{2}}^{2}\right\|+\operatorname{Re}\left(T_{\lambda} v, v\right)=\operatorname{Re}\left(\left\{I+T_{\lambda}\right\} v, v\right),
$$




$$
\|v\|_{L^{2}} \leq\left\|\left\{I+T_{\lambda}\right\} v\right\|_{L^{2}} \quad(\operatorname{Re} \lambda>0) .
$$

The inequalities (3.21) and (3.22) hold at $\operatorname{Re} \lambda \geq 0$ since $T_{\lambda}$ is continuous in $\lambda$ (The method of the proof of (3.22) is the same one as in Lemma 3, 4 of Mizohata and Mochizuki [2]). We now study the case $\operatorname{Re} \lambda \leq 0$.

$$
\text { (3.23) } \begin{aligned}
& \left\{T_{\lambda}-T_{\lambda-h}\right\} v \\
= & \left\{\lambda a(x) \cdot G_{1}(\lambda) \cdot a(x) \cdot-(\lambda-h) a(x) \cdot G_{1}(\lambda-h) \cdot a(x) \cdot\right\} v \\
= & {\left[h \cdot a(x) \cdot G_{1}(\lambda) \cdot a(x) \cdot+(\lambda-h) \cdot a(x)\left\{G_{1}(\lambda)-G_{1}(\lambda-h)\right\} a(x)\right] v . }
\end{aligned}
$$

Applying i) and ii) of Lemma 2 to (3.23) for sufficiently small $h \geq 0$ and $\operatorname{Re} \lambda-h \geq-\delta^{\prime}<0$, we have

(3. 24) $\left\|\left\{T_{\lambda}-T_{\lambda-l}\right\} v\right\|_{L^{2}}$

$$
\begin{aligned}
& \leq|h|\left(\sup _{x}\left|a(x) e^{+\delta^{\prime} x}\right|\right) \cdot\left|G_{1}(\lambda) a(x) v\right|_{-\delta} \\
& \quad+|\lambda-h|\left(\sup _{x} \mid a(x) e^{+\delta|x|}\right)\left|\left\{G_{1}(\lambda)-G_{1}(\lambda-h)\right\} a(x) v\right|_{-\delta} \\
& \leq \text { Const. }|h|(1+|\lambda|)^{-1}|a(x) \cdot v|_{\delta} \\
& \quad+\text { Const. }|\lambda-h| \cdot(1+|\lambda|)^{-1} \cdot|h| \cdot|a(x) \cdot v|_{\delta}
\end{aligned}
$$

$\leq$ Const. $|h|\|v\|_{L^{2}}, \quad$ for $\operatorname{Re} \lambda \geq 0$.

On the other hand,

$$
\|v\|_{L^{2}}^{2}+\operatorname{Re}\left(T_{\lambda} v, v\right)=\operatorname{Re}\left(\left\{I+T_{\lambda-h}\right\} v, v\right)+\operatorname{Re}\left(\left\{T_{\imath}-T_{\lambda-h}\right\} v, v\right) .
$$

Since $\operatorname{Re}\left(T_{\lambda} v, v\right) \geq 0$ from (3.21) when $\operatorname{Re} \lambda=0$,

$$
\|v\|_{L^{2}}^{2} \leq\left\|\left\{I+T_{\lambda-h}\right\} v\right\|_{L^{2}}\|v\|_{L^{2}}+\left\|\left\{T_{\lambda}-T_{\lambda-i}\right\} v\right\|_{L^{2}}\|v\|_{L^{2}} .
$$

Since $\left\|\left\{T_{\lambda}-T_{\lambda-h}\right\} v\right\|_{L^{2}} \leq(1 / 2)\|v\|_{L^{2}}$ for $|h| \leq \delta^{\prime \prime}$ from (3.24) if we choose sufficiently small $\delta^{\prime \prime}>0$, replacing $\lambda-h$ with $\lambda$, we have the estimate

$$
\|v\|_{L^{2}} \leq 2\left\|\left\{I+T_{\lambda}\right\} v\right\|_{L^{2}} \text { at } \operatorname{Re} \lambda \geq-\delta^{\prime \prime}<0 .
$$

Thus there exists the inverse $\left(I+T_{\lambda}\right)^{-1}$ at $\operatorname{Re} \lambda \geq-\delta^{\prime \prime}<0$, which satisfies

$$
\left\|\left(I+T_{\lambda}\right)^{-1} h(x)\right\|_{L^{2}} \leq 2\|h(x)\|_{L^{2}} .
$$

This implies the existence of the inverse of $\left\{I+\lambda G_{1}(\lambda) \cdot b(x) \cdot\right\}$ in $\mathcal{A}(\delta)$ at $\operatorname{Re} \lambda \geq-\delta^{\prime \prime}<0$ by (3.20). In fact 


$$
\left\{I+\lambda G_{1}(\lambda) \cdot b(x) \cdot\right\}^{-1}=-\lambda G_{1}(\lambda) \cdot a(x) \cdot\left(I+T_{\lambda}\right)^{-1} \cdot a(x) \cdot+I \in \mathcal{A}(\delta) .
$$

From (3.25) we obtain the estimate of norm of this inverse, that is,

$$
\begin{aligned}
\| & \left\{I+\lambda G_{1}(\lambda) \cdot b(x) \cdot\right\}^{-1} \|^{\|} \delta \\
& \leq\left|\lambda\left\|\left.G_{1}(\lambda)\right|_{\delta} \cdot \sup _{x}\left|e^{\delta|x|} a(x)\right| \cdot\right\|\left\{I+T_{\lambda}\right\}^{-1} \|_{L^{2}} \cdot \sup _{x}\right| e^{\delta_{|x|} \mid} a(x) \mid+1 \\
& \leq \text { Const. }<\infty .
\end{aligned}
$$

This proves (3.19).

When $\operatorname{Re} \lambda$ is sufficiently large, $G_{2}(\lambda)=\left\{I+\lambda G_{1}(\lambda) \cdot b(x)\right\}^{-1} \cdot G_{1}(\lambda)$ from (3.18). The right hand side of this equality can be continued to an analytic function in $\operatorname{Re} \lambda \geq-\delta^{\prime \prime}<0$ from (3.19) and Lemma 2. Applying the estimates of $i$ ) of Lemma 2 for $G_{1}(\lambda)$ and of (3.19) the estimate of Lemra 3 for $G_{2}(\lambda)$ follows.

The proof of Lemma 3 is complete.

\section{§. Proof of Theorem 1}

Let $u(x, t)$ be a solution for initial value problem (1.1), (1.2). And let $\tilde{u}(x, \lambda)$ be the Laplace image of $u(x, t)$ with respect to $t$ in the sence of $L^{2}$. Then by the inversion formula of Laplace transform for a positive constant $\sigma_{0}$,

$$
u(x, t)=\frac{1}{2 \pi i} \lim _{\tau \rightarrow \infty} \int_{\sigma_{0}-i \tau}^{\sigma_{0}+i \tau} e^{\lambda t} \tilde{u}(x, \lambda) d \lambda, \quad \text { in } L^{2}
$$

And $\tilde{u}(x, \lambda)$ is a solution in $L^{2}$ for the equation that

$$
\left\{-\Delta+c(x)+\lambda b(x)+\lambda^{2}\right\} \tilde{u}(x, \lambda)=\frac{f(x)}{\lambda-i \omega},
$$

that is,

$$
\tilde{u}(x, \lambda)=\frac{1}{\lambda-i \omega} G_{2}(\lambda) \cdot f(x) .
$$

If we regard $\tilde{u}(x, \lambda)$ as a function in $Q(-\delta)$, we can apply Lemma 3 to $\tilde{u}(x, \lambda)$. When $f(x)$ belongs to $Q(\delta), \tilde{u}(x, \lambda)$ is analytic in $\operatorname{Re} \lambda \geq-\delta^{\prime \prime}<0$ except for one point $\lambda=i_{\omega}$ which is a simple pole with the residue $G_{2}(i \omega) \cdot f(x)$ and, where $|\lambda|$ is large, $\tilde{u}(x, \lambda)$ satisfies the estimate

$$
\left\|e^{-\delta|x|} \tilde{u}(x, \lambda)\right\|_{L^{2}} \leq \text { Const. }\left(1+|\lambda|^{2}\right)^{-1}\left\|e^{\delta|x|} f(x)\right\|_{L^{2}} .
$$


This implies by means of Cauchy integral formula

$$
\begin{aligned}
u(x, t) & =\frac{1}{2 \pi i} \int_{\sigma-i \infty}^{\sigma+i \infty} e^{\lambda t} \tilde{u}(x, \lambda) d \lambda, \quad \text { in } Q(-\delta) \\
& =\frac{1}{2 \pi i} \int_{-\varepsilon-i \infty}^{-\varepsilon+\infty} e^{\lambda t} \tilde{u}(x, \lambda) d \lambda+G_{2}(i \omega) \cdot f(x) e^{i \omega t} \text {, in } Q(-\delta),
\end{aligned}
$$

where $\delta^{\prime \prime} \geq \varepsilon>0$.

In order to prove the rate of approach of Theorem 1 we require the stronger estimate of $G_{2}(\lambda) \cdot f(x)$ for $\lambda$ under the assumption to $f(x)$ in Theorem 1 .

Since $\Delta f \in Q(-\delta)$, we have

$$
\begin{aligned}
& G_{2}(\lambda) \cdot f(x)=\frac{f(x)-g(x, \lambda)}{\lambda^{2}}, \\
& g(x, \lambda)=G_{2}(\lambda) \cdot[\{\lambda b(x)+c(x)\} f(x)+\Delta f(x)] .
\end{aligned}
$$

Applying Lemma 3, for sufficiently large $|\lambda|$,

$$
\left\|e^{-\delta|x|} G_{2}(\lambda) \cdot f(x)\right\|_{L^{2}} \leq \text { Const. }(|\lambda|+1)^{-2}, \quad \text { at } \operatorname{Re} \lambda \geq-\delta^{\prime \prime}<0 .
$$

On the other hand, $V(x, \lambda)=G_{2}(\lambda) \cdot f(x)$ satisfies the equation

$$
\begin{aligned}
V(x, \lambda) & +\frac{1}{4 \pi} \int \frac{e^{-\lambda|x-y|}}{|x-y|} c(y) V(y, \lambda) d y \\
& +\frac{\lambda}{4 \pi} \int \frac{e^{-\lambda|x-y|}}{|x-y|} b(y) V(y, \lambda) d y=\frac{1}{4 \pi} \int \frac{e^{-\lambda|x-y|}}{|x-y|} f(y) d y .
\end{aligned}
$$

This implies that

$$
\begin{aligned}
& \sup _{x \in K}|V(x, y)| \\
& \leq \text { Const. }\left[\left\|e^{-\delta|x|} V(x, \lambda)\right\|_{L^{2}}+|\lambda|\left\|e^{-\delta|x|} V(x, y)\right\|_{L^{2}}\right. \\
& \left.\quad+(|\lambda|+1)^{-2}\left\|e^{\delta|x|}\{f(x)+\Delta f(x)\}\right\|_{L^{2}}\right],
\end{aligned}
$$

where $K$ is an arbitrary bounded set in $E^{3}$. In fact,

$$
\begin{aligned}
& \sup _{x \in K}\left|\frac{1}{4 \pi} \int \frac{e^{-\lambda|x-y|}}{|x-y|} p(y) d y\right| \\
& \quad \leq\left[\sup _{x \in K} \frac{1}{4 \pi} \int\left|\frac{e^{-\operatorname{Re} \lambda|x-y|} e^{-\delta^{\prime} y \mid}}{|x-y|}\right|^{2} d y\right] \cdot \int\left|e^{\delta|y|} p(y)\right|^{2} d y
\end{aligned}
$$

and

$$
\frac{1}{4 \pi} \int \frac{e^{-\lambda|x-y|}}{|x-y|} f(y) d y=\frac{1}{\lambda^{2}}\left\{f(x)-\frac{1}{4 \pi} \int \frac{e^{-\lambda \mid x-y}}{|x-y|} \Delta f(y) d y\right\} .
$$


Applying (4.2) to (4.4), we have

$$
\sup _{x \in K}|V(x, \lambda)| \leq \text { Const. }(|\lambda|+1)^{-1}, \quad \text { at } \operatorname{Re} \lambda \geq-\delta^{\prime \prime}<0 .
$$

Since $\tilde{u}(x, \lambda)=(\lambda-i \omega)^{-1} V(x, \lambda)$ and from (4.1)

$$
\begin{aligned}
& \sup _{\lambda \in K}\left|u(x, t)-G_{2}(i \omega) \cdot f(x) e^{i \omega t}\right| \\
& \quad \leq \sup _{x \in K}\left|\frac{1}{2 \pi i} \int_{-\varepsilon-i \infty}^{-\varepsilon+i \infty} e^{\lambda t} \frac{V(x, \lambda)}{\lambda-i \omega} d \lambda\right| \\
& \leq \frac{1}{2 \pi i} e^{-\varepsilon t} \int_{-i \infty}^{+i \infty}\left\{\sup _{x \in K} \mid V(x, \lambda-\varepsilon) !\right\}\left|\frac{1}{\lambda-\varepsilon-i \omega}\right| d \lambda \\
& \leq \text { Const. } e^{-\varepsilon t} \int_{-i \infty}^{+i \infty}(|\lambda|+1)^{-2} d \lambda, \quad \text { where } \varepsilon>0 \\
& =0\left(e^{-\varepsilon t}\right), \quad(t \rightarrow \infty) .
\end{aligned}
$$

This proves the rate of approach of Theorem 1 . Since $G_{2}(i \omega) \cdot f(x)$ $=V(x)$ satisfies (4.3) at $\lambda=i \omega$, it follows that $V(x)$ is a solution of (1.3) and Sommerfeld's radiation conditions.

The proof of Theorem 1 is complete.

\section{REFERENCES}

[1] Ladyzenskaja, O.A., On the principle of limiting amplitude, Uspehi Mat. Nauk, 12, No. 3 (1957), 161-164. (Russian)

[2] Mizohata, S. and K. Mochizuki, On the principle of limiting amplitude for dissipative wave equations, J. Math. Kyoto Univ. 6 (1966), 109-127.

[3] Eĩdus, D. M., On the principle of limiting absorption, Mat. Sb. 57 (1962), 13-44. (Russian)

[4] Povzner, A. Ja., On the expansion of arbitrary functions in characteristic functions of the operator $-\Delta u+c u$, Mat. Sb. 32 (1953), 109-156.

[5] Kiyama, S., On the exponential decay of solutions of the wave equation with the potential functions, Osaka J. Math. 4 (1967), 15-35.

[6] Morawetz, C. S., The limiting amplitude principle, Comm. Pure Appl. Math. 15 (1962), 349-361. 